\title{
The PMIPv6-Based Group Binding Update for IoT Devices
}

\author{
Jianfeng Guan, ${ }^{1}$ Ilsun You, ${ }^{2}$ Changqiao $\mathrm{Xu},{ }^{1}$ and Hongke Zhang ${ }^{3}$ \\ ${ }^{1}$ State Key Laboratory of Networking and Switching Technology, Beijing University of Posts and Telecommunications, \\ Beijing 100876, China \\ ${ }^{2}$ Department of Information Security Engineering, Soonchunhyang University, Asan-si 31538, Republic of Korea \\ ${ }^{3}$ National Engineering Laboratory for Next Generation Internet Interconnection Devices, Beijing Jiaotong University, \\ Beijing 100044, China \\ Correspondence should be addressed to Ilsun You; ilsunu@gmail.com
}

Received 1 October 2015; Accepted 17 January 2016

Academic Editor: Kamal Deep Singh

Copyright (C) 2016 Jianfeng Guan et al. This is an open access article distributed under the Creative Commons Attribution License, which permits unrestricted use, distribution, and reproduction in any medium, provided the original work is properly cited.

\begin{abstract}
Internet of Things (IoT) has been booming with rapid increase of the various wearable devices, vehicle embedded devices, and so on, and providing the effective mobility management for these IoT devices becomes a challenge due to the different application scenarios as well as the limited energy and bandwidth. Recently, lots of researchers have focused on this topic and proposed several solutions based on the combination of IoT features and traditional mobility management protocols, in which most of these schemes take the IoT devices as mobile networks and adopt the NEtwork MObility (NEMO) and its variants to provide the mobility support. However, these solutions are in face of the heavy signaling cost problem. Since IoT devices are generally combined to realize the complex functions, these devices may have similar movement behaviors. Clearly analyzing these characters and using them in the mobility management will reduce the signaling cost and improve the scalability. Motivated by this, we propose a PMIPv6based group binding update method. In particular, we describe its group creation procedure, analyze its impact on the mobility management, and derive its reduction ratio in terms of signaling cost. The final results show that the introduction of group binding update can remarkably reduce the signaling cost.
\end{abstract}

\section{Introduction}

According to the statistics analysis, the number of Internet of Things (IoT) devices is expected to reach up to 50 billion by 2020 [1]. Figure 1 shows several typical application scenarios of IoT, which consist of vehicle networks, Wireless Body Networks, and so on. Due to the large volumes of mobile IoT devices, it becomes a big challenge to provide well mobility support for IoT [2]. IPv6 is believed to be a suitable protocol [3] thanks to its large address space and specific mechanisms to support mobility, such as Mobile IPv6 (MIPv6) [4] and its potential solutions for mobility management.

However, these solutions are designed for portable devices such as cell phone or PC which have different application requirement from the IoT devices; therefore, they need to be improved or enhanced in terms of bandwidth, energy consumption, and scalability.

It is worth to note from such application scenarios that the group is one of important characters in IoT [5-7]. Therefore, several works have focused on this problem and proposed lots of solutions [8-20] most of which tried to apply the NEtwork MObility (NEMO) [21] into the IoT.

NEMO as a mobility support protocol for mobile network is derived from MIPv6 in which mobile router (MR) is introduced to deliver all the packets for mobile network nodes via the bidirectional tunnel between MR and its Home Agent (HA) [21]. When it is applied in IoT scenarios, the MR is generally used as the leader to perform the mobility signaling messages on behalf of all mobile network nodes. However, due to the frequent mobility, the mobile network nodes will change their attachment points dynamically which may introduce the additional signaling and transmission costs due to the nested tunnels operations according to standard NEMO protocol procedure.

In this work we focus on group characteristics of IoT devices while not only studying the dynamic group management mechanism, but also extending the bulk binding update 


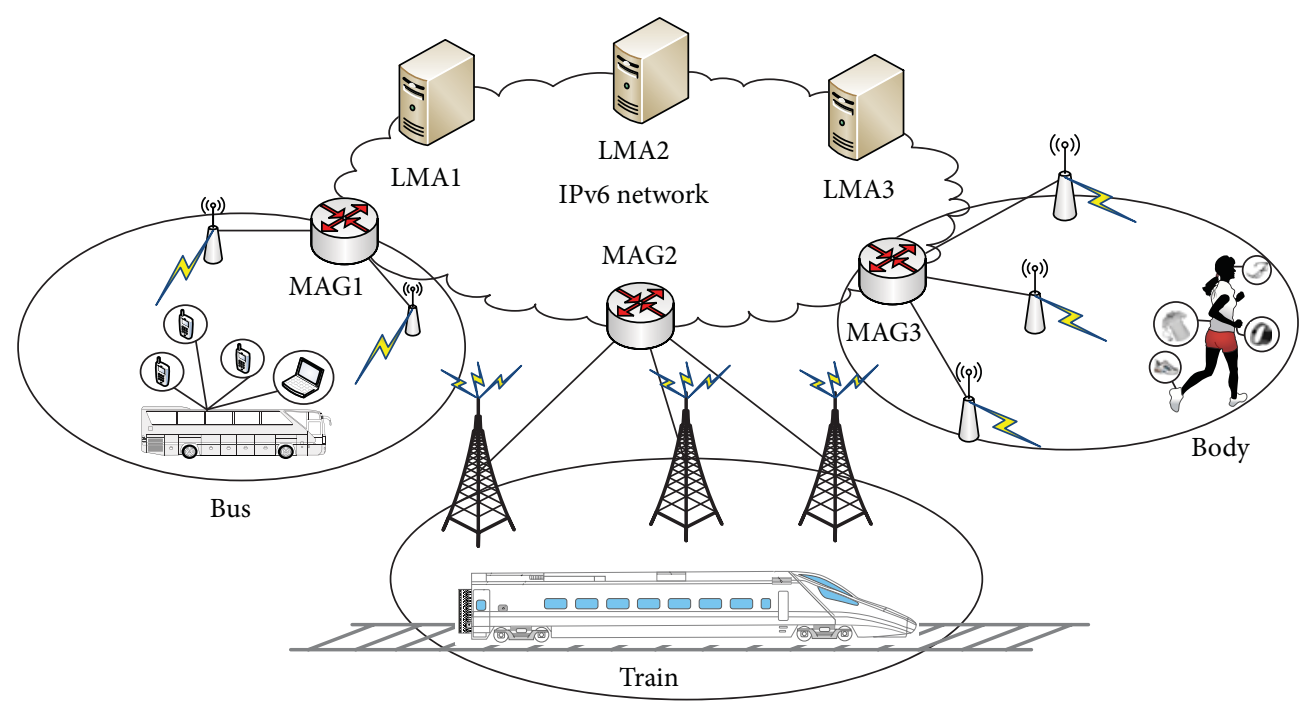

LMA: Local Mobility Anchor MAG: Mobile Access Gateway

FIgURE 1: Application scenarios of IoT.

of PMIPv6 [22] to set up the dynamic groups for IoT devices. The contributions of our work are summarized as follows:

(1) propose the group binding update scheme based on PMIPv6 to update a group of IoT devices to reduce the signaling cost;

(2) introduce the extensions for group identifier and the operation considerations of Mobility Access Gateway (MAG) and Local Mobility Anchor (LMA) and give the detailed operation flow of group binding update procedure;

(3) derive the signaling cost reduction ratio and analyze the relationship between group size and signaling cost.

The organization of this paper is shown as follows. Section 2 introduces the related works of IPv6 mobility management solutions and their applications in IoT. Section 3 describes the PMIPv6-based group binding update solution in detail. Section 4 models and analyzes the impact of group binding update of PMIPv6, followed by deriving the cost reduction ratio and giving the numerical results. Finally, Section 5 concludes our work.

\section{Related Work}

2.1. MIPv6 and Extensions for IoT. MIPv6 is a basic mobility support solution, which introduces the HA to perform the handover and location managements. In MIPv6, the HAs forward the packets via the bidirectional tunnels for each registered mobile node $(\mathrm{MN})$ to maintain the ongoing sessions. MIPv6 introduces two kinds of address, Home Address (HoA) and Care-of Address (CoA), which are used to separate identifier functions and routing functions of IP address.
In specific, HoA is the permanent identifier of $\mathrm{MN}$ used to set up the upper layer sessions, while CoA is the location identifier used to transmit the packets. Compared with MIPv4, MIPv6 also provides optimal routing process by maintaining the binding cache of each $\mathrm{MN}$ in Corresponding Node $(\mathrm{CN})$. Hierarchical MIPv6 (HMIPv6) [23] was further proposed to solve the signaling cost problem in MIPv6 caused by the frequent Ping-Pong movements between two adjacent subnets, in which the Mobility Anchor Point (MAP) is introduced to deal with the micromobility on behalf of HA. Besides, fast handover for MIPv6 (FMIPv6) [24] was also proposed to reduce the handover delay with the assist of L2 trigger, which supports preconfiguration via Handover Indication (HI) and Handover Acknowledgment (HAck) messages to reduce the packet loss by setting up a short-time bidirectional tunnel between the previous attachment and new attachment points. However, these improved solutions require the involvements of MNs which may result in the excessive energy consumption for the power limited mobile devices and also restrict the wide deployment due to the additional function extensions.

Proxy MIPv6 (PMIPv6) [25] was proposed to deal with the mobility-related signaling messages. In particular, PMIPv6 introduces the MAG on behalf of MNs and emulate the home link for its attached MNs by setting up the bidirectional tunnel between the serving MAG and its LMA. Some works $[26,27]$ have testified that the PMIPv6 can reduce the handover delay and signaling cost greatly, and it is easy to deploy. Besides, some solutions such as F-PMIPv6 [28] were proposed by combining PMIPv6 and FMIPv6 to achieve better performance.

The above analyses show that the PMIPv6 and its variants are more suitable for mobile devices. However, these solutions are designed for traditional mobile devices such as smartphones, mobile Internet devices with the objective to provide 
the continual connections for MNs, which is different from the requirements of the IoT devices. For example, the IoT session mobility is not always required [29]. Therefore, to provide the mobility management for IoT devices, some IoT characteristics should be considered, such as limited computation capacity, limited network resource, and movement behaviors. Considering many IoT devices having different movement trajectory in different application scenarios such as Intelligent Transportation Systems (ITSs), Internet of Cars, and Wireless Body Networks, it becomes very important to analyze the group character to optimize the mobility management [30].

2.2. NEMO and Extensions for IoT. NEMO is a basic solution to provide the group mobility support. However, this solution will bring in high packet delivery cost with the increase of nodes and multiple nests of mobile networks. Therefore, several improved solutions have been proposed in the past few years.

2.2.1. FMIPv6-Based Schemes. FMIPv6-based schemes were proposed by combining FMIPv6 and NEMO, such as fNEMO [8, 9]. After that, EfNEMO [10] was proposed by adopting the tentative binding update which pre-registers a new address assigned by Next Access Router (NAR) for the MR with the HA before the layer- 2 association. The tentative binding update sets up a path between NAR and HA to deliver the traffic without the additional encapsulation. Therefore, it can reduce the handover latency and also reduce the traffic delivery cost thanks to the tentative binding update. This idea is similar to the transient binding for PMIPv6 [11], which can mitigate the tunneling burden and handover latency. However, they lack the consideration of dynamic character of mobile networks and are inefficient to deal with the dynamic of the mobile network nodes.

2.2.2. PMIPv6-Based Schemes. PMIPv6-based solutions aim to extend the PMIPv6 to support the network mobility. The early solution was proposed by Soto et al. [12] called NEMOenabled PMIPv6 (N-PMIPv6), which takes the MR as the mobile MAG (mMAG). However, it just simply combines the PMIPv6 and NEMO without the consideration of the character of IoT devices. After that, Jeon and Kim [13] employed a relay station to mitigate the packet tunneling costs, which is called proxy router-based NEMO (PRNEMO) that introduces the proxy router instead of the MR as forwarder between MAG and MNN to reduce the cost.

Besides, Lee et al. [14] also proposed the PMIPv6-based NEMO (P-NEMO) scheme which takes $\mathrm{MR}$ as $\mathrm{MN}$ and extends MAG and LMA to maintain mobile network prefix (MNP) for each MR and advertises MNP to mobile network nodes via Router Advertisement (RA) message. Furthermore, they also proposed the Fast P-NEMO (FP-NEMO) to improve the handover delay by combining P-NEMO with fast handover of PMIPv6. In vehicular networks, Kim and Lee [15] proposed a group-based fast handover by extending PMIPv6-based NEMO with the assist of bulk binding to reduce the overhead, in which the bulk binding update is used to perform the binding update and revocation operations on behalf of a group of mobility sessions [22]. Some detail operations of this solution are still under the way. For example, it requires the neighboring MAG information to set up the tunnels in advance which are only applied in some specific scenarios. This idea absorbs the advantages of both PMIPv6 and FMIPv6, which can provide better mobility management for mobile networks. However, they are still limited by NEMO, which cannot support the mobile network nodes efficiently.

2.2.3. DMM-Based Schemes. DMM-related schemes apply MIPv6, PMIPv6, and NEMO in a distributed way. Based on the MIPv6, Sornlertlamvanich et al. [16] distributed the HA functions to each access router and set it as the default gateway of each attached mobile network. Besides, based on the PMIPv6, Do and Kim [17] combined the LMA and MAG functions into each access router and introduced a proxy router to manage MNs' mobility and set up a central session database to track MNs and the proxy router. Furthermore, Ernest et al. [18] improved these schemes by decomposing the logical functions of LMA to location management and resource management. It not only extends LMA to support the home network prefix allocation functions, but also extends MR to support the Delegating Router (DR) and Request Router (RR) functions. More recently, Nguyen and Bonnet proposed the H-NEMO solution which combines the centralized mobility management with the distributed mobility management [19]. This new solution assigns two prefixes for Mobile Anchor Router (MAR) called MAR-prefix and central mobility anchor called LMA-prefix and uses them for short-lived flow and long-lived flow, respectively. The DMM-based solutions can improve the scalability of the mobility scenarios with high session density; however, as for IoT devices, the session mobility is not always necessary.

2.2.4. ID/Locator Separation Based Schemes. Another solution is based on the splitting of identifier and location. Ishino et al. [29] proposed a routing-based mobility management which realized the routing aggregation by adopting the Bloom Filter to store routing information. This scheme separated the identifier and locator and provided the identifier routing based on Bloom Filter to support the mobility. Besides, Kim et al. [20] also suggested adopting the ID/locator separation to provide the network mobility, in which MR sends an aggregate location update message on behalf of the whole mobile network to reduce the signaling cost and handover delay. This kind of solution requires large modification of the existing network architecture, which cannot be easily deployed.

2.3. Group Mobility Management for IoT. Group-based approaches have been proposed to improve the mobility. Based on the movement trace similarity, Fu et al. [5] proposed a group mobility management mechanism, in which the global location database divides the nodes into different groups. The group leader performs the mobility management on behalf of the other group members. Moreover, Galluccio et al. [31, 32] derived an analytical framework to assess the impact of object group mobility when adopting MIPv6. 
Based on the above analysis, we find these solutions to have the following characters:

(1) IoT devices are power limited and should support the bulk operations to reduce the cost to reduce the redundancy operations.

(2) NEMO-based solution defines a group by mobile network prefix and provides the mobility support via mobile router.

(3) The group-based method is dynamic, and the group is temporarily set up to realize the aggregative registrations. In another word, the group-based method can be viewed as a general case of NEMO.

In this paper, we propose a group binding update scheme based on PMIPv6 to update a group of IoT devices to reduce the signaling cost and analyze its impact on signaling cost.

\section{PMIPv6-Based Group Binding Update Solution}

The proposed scheme is based on the PMIPv6 bulk binding update mechanism which is designed to optimize the binding update and revocation operations for a group of mobility sessions by introducing group identifier. The group identifier can be assigned by MAG or LMA and will be exchanged via Proxy Binding Update (PBU) and Proxy Binding Acknowledgment (PBA) messages with " $B$ " flag and is finally recorded in the Binding Cache Entry (BCE) of LMA. Therefore, the bulk binding is generally used to extend the lifetimes of multiple mobility sessions and revoke all the sessions hosted on the failed service card.

The proposed scheme extends the bulk binding not for session groups but for the node groups and its basic idea is to set up a group for IoT devices based on some metrics such as the movement similarity [5] or administrative domain $[6,7]$ to perform the binding update in form of node groups. By this way, the signaling cost therefore will be reduced. To provide this group binding update, we have to extend the mobile node group identifier option, binding information on MAG and LMA, and design the group creation procedure of IoT devices.

3.1. Mobile Node Group Identifier Option Extensions. The mobile node group identifier option (MNGIO) defined in [22] is used to carry the group identifier. Figure 2 shows the extended MNGIO format.

(i) The type field is 50 which has been assign by IANA to represent that this is a mobile node group identifier option.

(ii) The length field is 6 bytes which exclude the type and length field.

(iii) The subtype field is 8 -bit which has 256 types. While the values of 0 and 255 have been reserved, the value of 1 has been assigned to bulk binding update group. In our work, we introduce a new subtype called group binding update and temporarily set its value of 2 for IoT devices group binding update.

\begin{tabular}{|c|c|c|c|}
\hline & & & \\
\hline $\begin{array}{l}\text { Type } \\
\text { (8-bit) }\end{array}$ & $\begin{array}{l}\text { Length } \\
\text { (8-bit) }\end{array}$ & $\begin{array}{r}\text { Subtype } \\
\text { (8-bit) }\end{array}$ & $\begin{array}{c}\text { Reserved } \\
\text { (8-bit) }\end{array}$ \\
\hline \multicolumn{4}{|c|}{$\begin{array}{l}\text { Mobile node group identifier } \\
\text { (32-bit) }\end{array}$} \\
\hline
\end{tabular}

Figure 2: Mobile node group identifier option.

\begin{tabular}{|l|c|c|cc|}
\multicolumn{1}{r}{8} & \multicolumn{1}{r}{16} & 24 \\
\hline$T$ & $P$ & $\begin{array}{c}\text { Reserved } \\
\text { (6-bit) }\end{array}$ & \multicolumn{2}{c|}{$\begin{array}{c}\text { Identifier } \\
\text { (24-bit) }\end{array}$} \\
\hline
\end{tabular}

$$
\begin{aligned}
& T=1 \text { indicates a group ID assigned by LMA } \\
& T=0 \text { indicates a group ID assigned by MAG } \\
& P=1 \text { indicates a permanently assigned ("well-known") group ID } \\
& P=0 \text { indicates a dynamically assigned group ID }
\end{aligned}
$$

FIGURE 3: Extended group identifier format.

(iv) The mobile node group identifier (noted as G-ID) is 32-bit, which can be assigned by MAG and LMA. The all 0 and all 1 are reserved.

Figure 3 shows the extended G-ID format which is 4 octets and divides into flag and identifier fields. The first 1 octet is set to different flags, in which we introduce two flags " $T$ " and " $P$ " and reserve 6 bits for future extensions.

“ $T$ " flag distinguishes the assigner of group ID. $T=1$ indicates a group ID assigned by LMA (called LG-ID), and $T=0$ indicates a group ID assigned by MAG (called MG-ID). More specifically, MG-ID represents the group of IoT devices that attaches to the same MAG, while LG-ID represents the group of IoT devices with the same HA or LMA. In the proposed scheme, LG-ID is predefined by HA or LMA to divide its IoT devices into different groups in advance, while MG-ID is used to record the attached IoT devices in the same access network. " $P$ ” flag indicates the well-known group ID. $P=1$ indicates a permanently assigned group ID, while $P=0$ indicates a transient or dynamical group ID. The following 3 octets identify different groups in which all zeros and all ones are revised.

3.2. MAG and LMA Operations Extensions. MAG extends its Binding Update List (BUL) to add the MG-ID and LG-ID fields, while LMA extends its BCE to include MG-ID and LGID. LMA predefines the groups of IoT devices and assigns a LG-ID for each group in advance, so that the IoT device in the same group will be marked by LG-ID in the BCE of LMA. MAG creates the groups of IoT devices dynamically according to the group policy (e.g., movement-based method [5]), assigns a MG-ID for each group, and records the MGID in the Binding Update List for each node in that group. LMA and MAG exchange LG-ID and MG-ID via PBU and PBA messages within the MNGIO. Based on (MG-ID, LGID) information, both MAG and LMA update their Binding Update List and binding cache, respectively. Similar to [22], the extensions of BCE and BUL use the MAG bulk binding update group ID to record the MG-ID received from MAG 


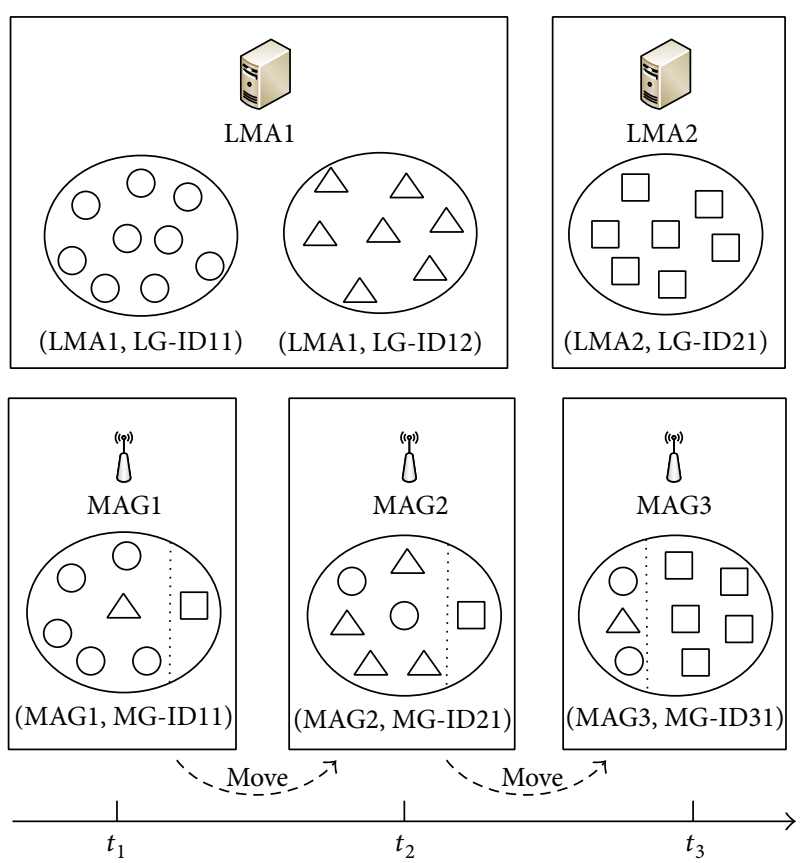

FIgURE 4: The IoT group creation procedure.

and use the LMA bulk binding update group ID to record the LG-ID received from LMA.

3.3. IoT Group Creation Procedure. We adopt Figure 4 to describe the IoT group creation procedure. Assume that there are three groups of IoT devices called LG-ID11, LG-ID12, and LG-ID21 initially. LG-ID11 and LG-ID12 are assigned by LMA1, and LG-ID21 is assigned by LMA2. Therefore, we can note them as (LMA1, LG-ID11), (LMA1, LG-ID12), and (LMA2, LG-ID21).

At $t_{1}$, several IoT devices attach to MAGl. Based on the movement trace character or other grouping policies, MAG1 sets up a group and assigns a group identifier MG-ID11. After exchanging the group information between MAG and LMA, the devices in MG-ID1 can be further divided into multiple subgroups based on their LMAs. As shown in Figure 4, the MG-ID11 group can be divided into two parts. The left part is the subgroup of devices belonging to LMA1, while the right part belongs to LMA2. The binding update will be performed in form of a group with same (MG-ID, LG-ID). To this end, the devices in the same subgroup belonging to LMA1 will only perform once registration with their LMA, while the other IoT devices in the group have to perform multiple registrations with their LMAs, respectively. By this way, the signaling cost can be greatly reduced especially when the devices' density is high. Once these IoT devices move into another access network, a new group will be created and perform similar procedure as $t_{1}$. By setting up the group, the IoT devices belonging to the same LMA will only update once with their LMAs, and therefore the signaling cost will be reduced.

3.4. Mobility Management Procedure. Assume that the LGIDs are assigned by LMA in advance and stored in LMA's
BCEs. Figure 5 shows the signaling flow of group binding update when the IoT devices enter the PMIPv6 domain, and its detailed steps are shown as follows.

(1) In the beginning, MAG1 detects the attachment events of IoT devices to acquire MN-IDs and their profiles. After that, MAG1 divides the attached IoT devices into different groups based on the grouping policy such as movement similarity and assigns the MG-IDs for these groups. MAG1 records the group members of each IoT group and updates the related BUL with MG-ID for each group member.

(2) IoT device (noted as MN1) belonging to MG-ID1 sends Router Solicitation message (noted as RS1) to MAG1 at any time after it attached to MAG1.

(3) After receiving RS1, MAG1 sends PBU message with " $B$ " flag to MN1's LMA (noted as LMA1). The PBU message carries the MN1's ID (MN-ID1) and group ID (MG-ID1).

(4) Once LMA1 receives this PBU message, it will update the related $\mathrm{BCE}$ based on the MN-ID and update the MG-ID field, and then it will reply with a PBA message with " $B$ " flag in which MN1's LG-ID1 is carried in the MNGIO field.

(5) Once MAG1 receives PBA, it will update the related BUL with the LG-ID1. In this way, the group information is exchanged.

(6) In a similar way, other IoT devices (such as MN2 and MN3) perform a similar binding update procedure.

(7) After finishing the initiation procedure, the MAG will perform the group binding update and update the group binding relationship of MG-ID1. For all the nodes with the same LMA, it only performs one binding update procedure.

(8) In a similar operation, MAG2 and MAG3 will perform the same procedure as MAG1. By this way, the IoT devices in the same group such as MG-ID and LG-ID will only perform once, and the overall binding update cost will be reduced.

\section{Model and Analysis}

We consider an IoT application scenario which consists of several IoT devices with different access technologies. Based on [5], we assume that an IoT device joins the group following a Poisson process with $\lambda$, and the dwell time of this IoT device in this group follows a general distribution with mean $1 / \mu$. Based on the $M / G / \infty$ queuing model, the steady-state probability for the number $M$ of IoT devices in this group MG-ID1 is

$$
P_{r}[M=x]=\frac{\alpha^{x} e^{-\alpha}}{x !},
$$

where $\alpha=E[M]=\lambda / \mu$.

We denote by $C_{\Delta}$ as unit binding update cost of mobility management protocol and by $C_{o}$ as the binding update cost of original mobility management protocol. 


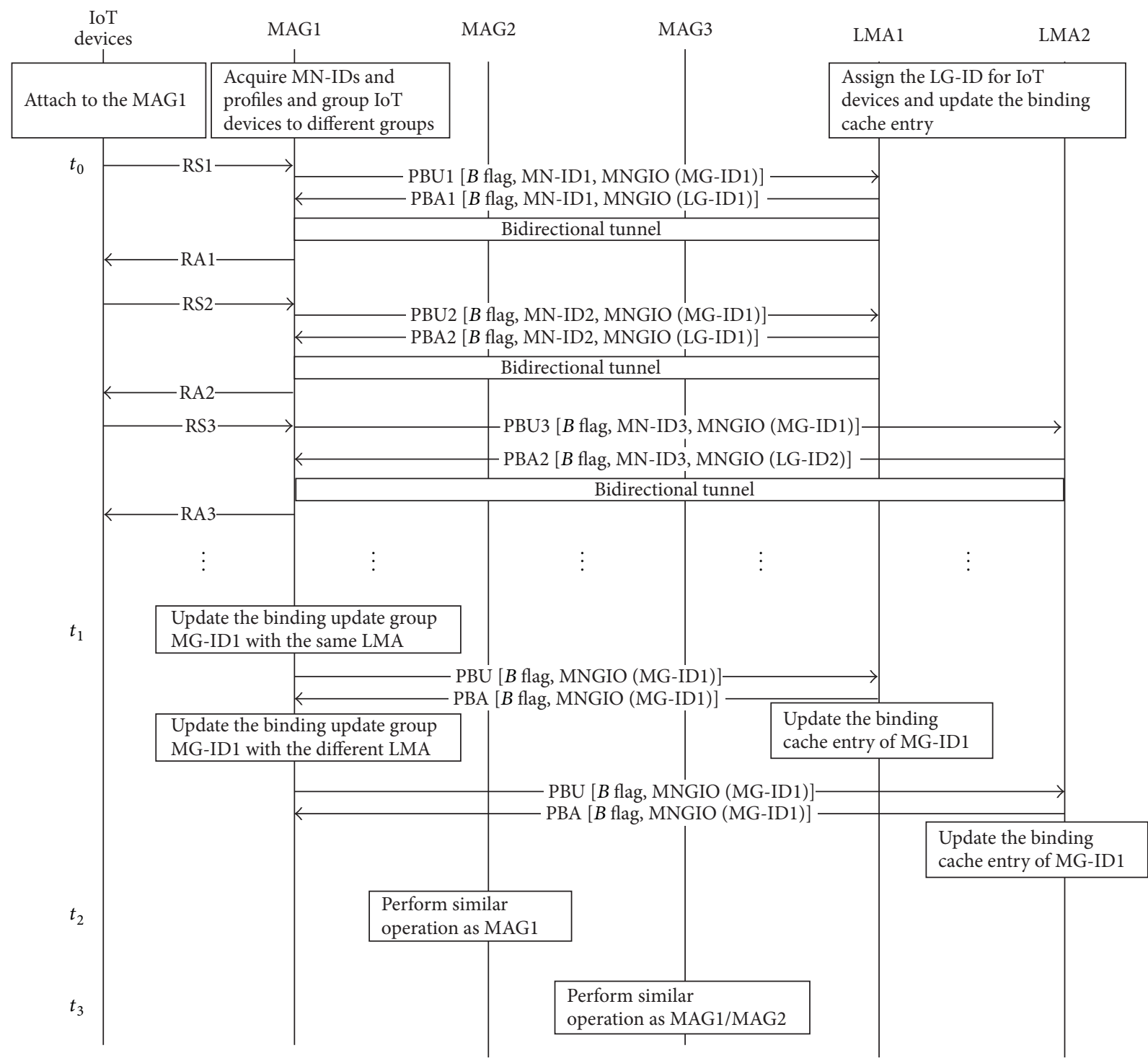

FIGURE 5: Group binding update operations for IoT devices.

To simplify the analysis, we divide the MG-ID into two subgroups and denote by $C_{1}$ as the binding update cost of subgroup in MG-ID with the same LMA and by $C_{2}$ as the binding update cost of IoT devices in MG-ID with different LMAs. Suppose that the probability of IoT devices located in the given group with the same LMA is $\rho$; we can get the cost of basic PMIPv6 and group binding as follows.

(1) Basic Binding Update Cost. For basic PMIPv6, the total update cost of $x$ nodes is shown as follows:

$$
C_{o \mid M=x}=x C_{\Delta} .
$$

Then, we can get its expected value as follows:

$$
E\left[C_{o}\right]=\sum_{x=1}^{\infty} x C_{\Delta} P_{r}[M=x]=E[M] C_{\Delta} .
$$

(2) Group Binding Update Cost. For the IoT devices in the group with the same LMA, they only perform once binding update, so the cost of group mobility management cost can be expressed as

$$
C=\rho C_{1}+(1-\rho) C_{2}
$$

Then, the expected value of $C$ is

$$
\begin{aligned}
E(C) & =E\left(\rho C_{1}+(1-\rho) C_{2}\right) \\
& =\rho E\left(C_{1}\right)+(1-\rho) E\left(C_{2}\right) .
\end{aligned}
$$

Next, we can get the

$$
\begin{aligned}
E\left[C_{1}\right] & =\sum_{x=1}^{\infty} C_{\Delta} P_{r}[M=x]=C_{\Delta}\left(1-P_{r}[M=0]\right) \\
& =C_{\Delta}\left(1-e^{-\alpha}\right) .
\end{aligned}
$$




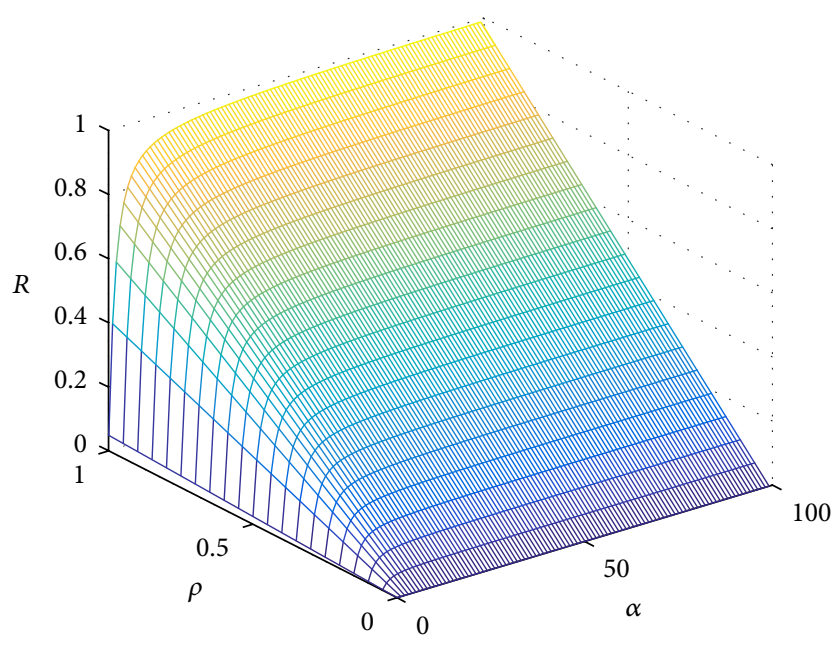

FIgURE 6: The reduction ratio of signaling cost.

As for the IoT devices in the group with different LMAs, they have to perform multiple binding update procedures, which can be expressed as follows:

$$
E\left[C_{2}\right]=\sum_{x=1}^{\infty} x C_{\Delta} P_{r}[M=x]=C_{\Delta} E[M] .
$$

Based on (6) and (7), the group-based signaling cost is

$$
E(C)=\rho\left(1-e^{-\alpha}\right) C_{\Delta}+(1-\rho) \alpha C_{\Delta} .
$$

To evaluate the performance of group binding update, we adopt the cost reduction ratio, which is shown as

$$
R=\frac{E\left(C_{o}\right)-E(C)}{E\left(C_{o}\right)}=\rho\left(\frac{e^{-\alpha}}{\alpha}-\frac{1}{\alpha}+1\right) .
$$

Figure 6 shows the reduction ratio according to (9), and Figure 7 shows the signaling cost reduction ratio under different group sizes and probabilities. We can get that, with the increase of the group size, the $R$ is increased and approaches to the $\rho$. With the increase of the $\rho$, the $R$ is also increased. These results show that with the increase of number of IoT devices with the same LMA, the signaling cost will be reduced greatly.

\section{Conclusions}

To provide effective mobility management for large-scale IoT devices, new behavior features should be taken into consideration. In this paper, we have studied the IoT mobility management challenges and proposed a group binding updating scheme based on the PMIPv6 for making the best use of the group character of IoT. In particular, we extend the PMIPv6 by introducing the group identifier to support the group binding to reduce the signaling cost, and a complete operation flow is described in detail. The analytical results show that our proposed group binding can reduce the signaling cost and the group impact. The further work is to improve the analytical model and derive it in more complex network conditions.

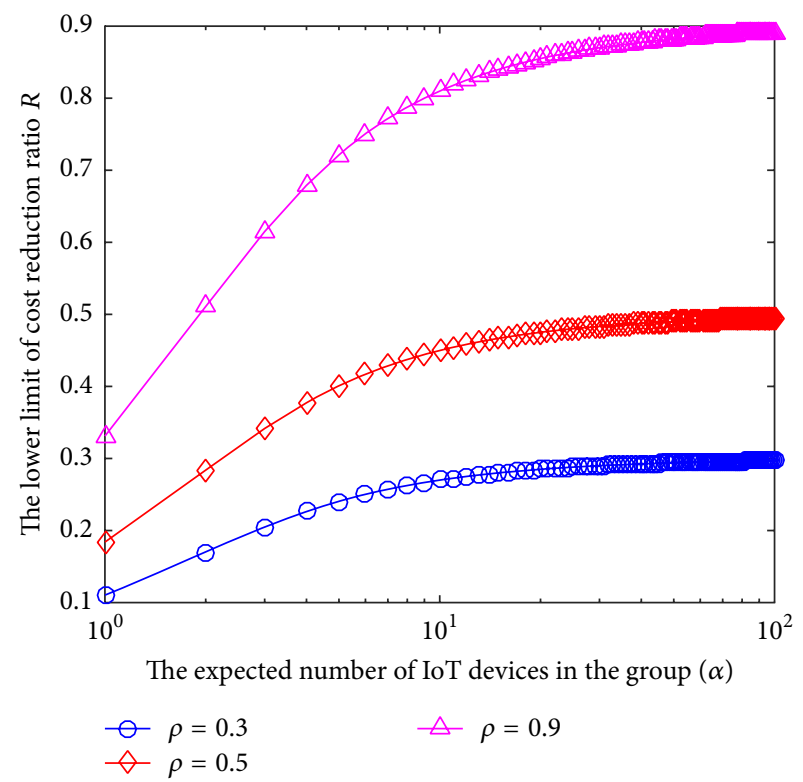

FIGURE 7: Impact of group size on the cost reduction.

\section{Conflict of Interests}

The authors declare that there is no conflict of interests regarding the publication of this paper.

\section{Acknowledgments}

This work was partially supported by the National Basic Research Program of China (973 Program) under Grant no. 2013CB329102, and also in part supported by Soonchunhyang University Research Funding.

\section{References}

[1] D. Evans, "The internet of things: how the next evolution of the internet is changing everything," Cisco IBSG-White Paper, 2011.

[2] H. Zhang, P. Dong, W. Quan, and B. Hu, "Promoting efficient communications for high speed railway using smart collaborative networking," IEEE Wireless Communications, vol. 22, no. 6, pp. 92-97, 2015.

[3] T. Savolainen, J. Soininen, and B. Silverajan, "IPv6 addressing strategies for IoT," IEEE Sensors Journal, vol. 13, no. 10, pp. 35113519, 2013.

[4] C. Perkins, D. Johnson, and J. Arkko, "Mobility support in IPv6," IETF RFC 6275, 2011.

[5] H.-L. Fu, P. Lin, H. Yue, G.-M. Huang, and C.-P. Lee, "Group mobility management for large-scale machine-tomachine mobile networking," IEEE Transactions on Vehicular Technology, vol. 63, no. 3, pp. 1296-1305, 2014.

[6] X. Jiang and D. H. C. Du, "BUS-VANET: a BUS vehicular network integrated with traffic infrastructure," IEEE Intelligent Transportation Systems Magazine, vol. 7, no. 2, pp. 47-57, 2015.

[7] X. Jiang, X. Cao, and D. H. C. Du, "Multihop transmission and retransmission measurement of real-time video streaming over DSRC devices," in Proceedings of the 15th IEEE International Symposium on a World of Wireless, Mobile and Multimedia 
Networks (WoWMoM '14), pp. 1-9, IEEE, Sydney, Australia, June 2014.

[8] L. Zhong, F. Liu, X. Wang, and Y. Ji, "Fast handover scheme for supporting network mobility in IEEE 802.16e BWA system," in Proceedings of the International Conference on Wireless Communications, Networking and Mobile Computing (WiCOM '07), pp. 1757-1760, Shanghai, China, September 2007.

[9] B. A. Mohammed and T. C. Wan, "Modified fast-integrated lightNEMOv6 handoff in IEEE 802.16e BWA networks," in Proceedings of the 2nd International Conference on Network Applications, Protocols and Services (NETAPPS '10), pp. 182-187, Alor Setar, Malaysia, September 2010.

[10] S. Ryu, K.-J. Park, and J.-W. Choi, "Enhanced fast handover for network mobility in intelligent transportation systems," IEEE Transactions on Vehicular Technology, vol. 63, no. 1, pp. 357-371, 2014.

[11] M. Liebsch, A. Muhanna, and O. Blume, "Transient binding for proxy mobile IPv6," RFC 6058, 2011.

[12] I. Soto, C. J. Bernardos, M. Calderon, A. Banchs, and A. Azcorra, "NEMO-enabled localized mobility support for internet access in automotive scenarios," IEEE Communications Magazine, vol. 47, no. 5, pp. 152-159, 2009.

[13] S. Jeon and Y. Kim, "Cost-efficient network mobility scheme over proxy mobile IPv6 network," IET Communications, vol. 5, no. 18, pp. 2656-2661, 2011.

[14] J.-H. Lee, T. Ernst, and N. Chilamkurti, "Performance analysis of PMIPv6-based network mobility for intelligent transportation systems," IEEE Transactions on Vehicular Technology, vol. 61, no. 1, pp. 74-85, 2012.

[15] M.-S. Kim and S. Lee, "Group-based fast handover for PMIPv6based network mobility in vehicular networks," in Proceedings of the IEEE Conference on Computer Communications Workshops (INFOCOM WKSHPS '15), pp. 113-114, IEEE, Hong Kong, April-May 2015.

[16] P. Sornlertlamvanich, S. Kamolphiwong, R. Elz, and P. Pongpaibool, "NEMO-based distributed mobility management," in Proceedings of the 26th IEEE International Conference on Advanced Information Networking and Applications Workshops (WAINA '12), pp. 645-650, IEEE, Fukuoka, Japan, March 2012.

[17] T.-X. Do and Y. Kim, "Distributed network mobility management," in Proceedings of the 5th International Conference on Advanced Technologies for Communications (ATC '12), pp. 319322, IEEE, Hanoi, Vietnam, October 2012.

[18] P. P. Ernest, H. A. Chan, O. E. Falowo, L. A. Magagula, and S. Céspedes, "Network-based distributed mobility management for network mobility," in Proceedings of the IEEE 11th Consumer Communications and Networking Conference (CCNC '14), pp. 417-425, IEEE, Las Vegas, Nev, USA, January 2014.

[19] T.-T. Nguyen and C. Bonnet, "A hybrid centralized-distributed mobility management architecture for network mobility," in Proceedings of the IEEE International Symposium on a World of Wireless, Mobile and Multimedia Networks (WOWMOM '15), pp. 1-9, Boston, Mass, USA, June 2015.

[20] Y. Kim, H. Ko, and S. Pack, "Network mobility support in distributed ID/locator separation architectures," in Proceeding of IEEE 11th Consumer Communications and Networking Conference (CCNC '14), pp. 521-523, Las Vegas, Nev, USA, January 2014.

[21] V. Devarapalli, R. Wakikawa, A. Petrescu, and P. Thubert, "Network mobility (NEMO) basic support protocol," IETF RFC 3963, 2005.
[22] F. Abinader, S. Gundavelli, K. Leung, S. Krishnan, and D. Premec, "Bulk binding update support for proxy mobile IPv6," RFC 6602, 2012.

[23] H. Soliman, C. Castelluccia, K. El Malki, and L. Bellier, "Hierarchical mobile IPv6 mobility management (HMIPv6)," RFC 4140, 2005.

[24] R. Koodli, Ed., Fast Handover for Mobile IPv6, RFC 4068, 2005.

[25] S. Gundavelli, K. Leung, V. Devarapalli, K. Chowdhury, and B. Patil, "Proxy mobile IPv6," IETF RFC 5213, 2008.

[26] J. Guan, H. Zhou, W. Xiao, Z. Yan, Y. Qin, and H. Zhang, "Implementation and analysis of network-based mobility management protocol in WLAN environments," in Proceedings of the International Conference on Mobile Technology, Applications, and Systems (Mobility '08), ACM, Yilan, Taiwan, September 2008.

[27] J. Guan, H. Zhou, Z. Yan, Y. Qin, and H. Zhang, "Implementation and analysis of proxy MIPv6," Wireless Communications and Mobile Computing, vol. 11, no. 4, pp. 477-490, 2011.

[28] H. Yokota, K. Chowdhury, R. Koodli, B. Patil, and F. Xia, "Fast handovers for proxy mobile IPv6," RFC 5949, 2010, http://tools .ietf.org/html/rfc5949.

[29] M. Ishino, Y. Koizumi, and T. Hasegawa, "A study on a routingbased mobility management architecture for IoT devices," in Proceedings of the 22nd IEEE International Conference on Network Protocols (ICNP '14), pp. 498-500, IEEE, Raleigh, NC, USA, October 2014.

[30] 3rd Generation Partnership Project (3GPP), “3rd Generation Partnership Project; technical specification group services and system aspects; service requirements for Machine-Type Communications (MTC); stage 1 (Release 11)," Tech. Rep. 3G TS 22.368, Sophia-Antipolis Cedex, Paris, France, 2011.

[31] L. Galluccio, G. Morabito, and S. Palazzo, "On the potentials of object group localization in the internet of things," in Proceedings of the IEEE International Symposium on a World of Wireless, Mobile and Multimedia Networks (WoWMoM '11), Lucca, Italy, June 2011.

[32] S. D’Oro, L. Galluccio, G. Morabito, and S. Palazzo, "Exploiting object group localization in the internet of things: performance analysis," IEEE Transactions on Vehicular Technology, vol. 64, no. 8, pp. 3645-3656, 2015. 

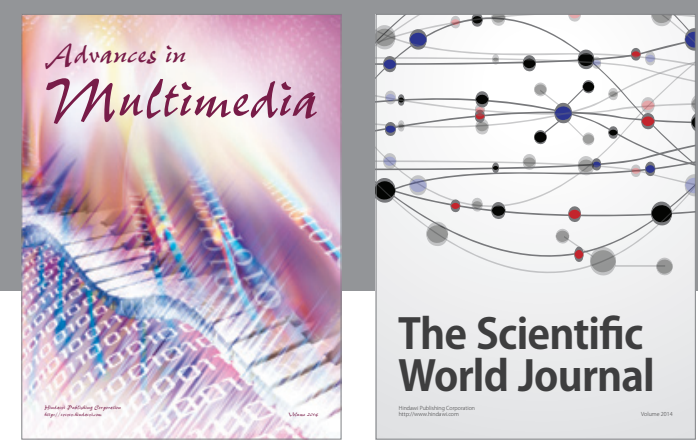

The Scientific World Journal
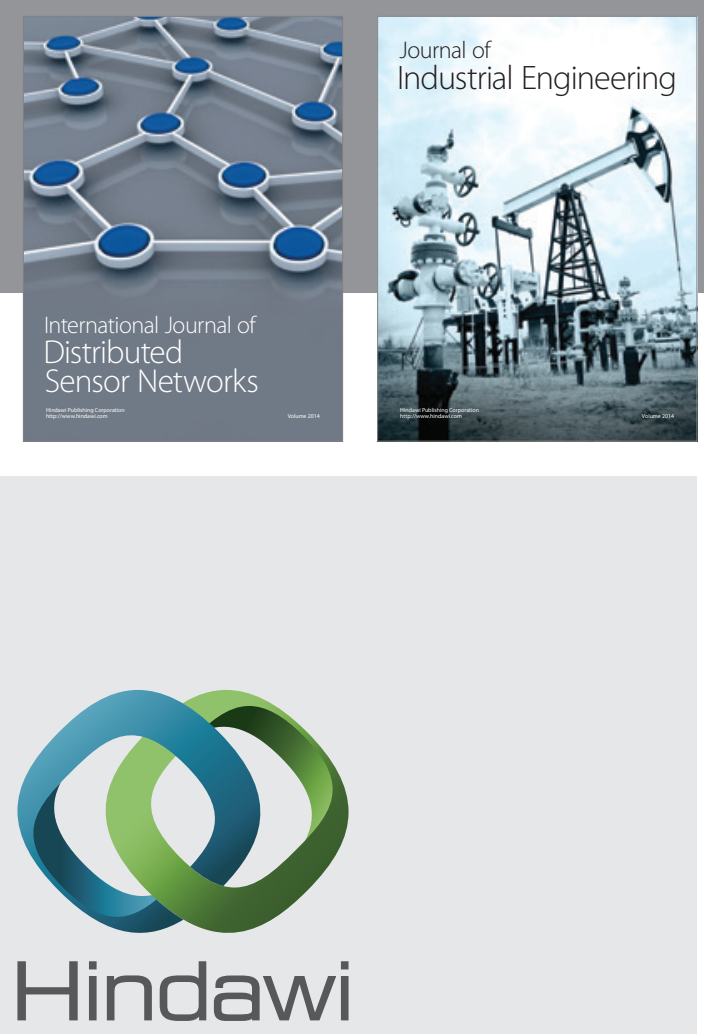

Submit your manuscripts at

http://www.hindawi.com

\section{Computer Networks} and Communications
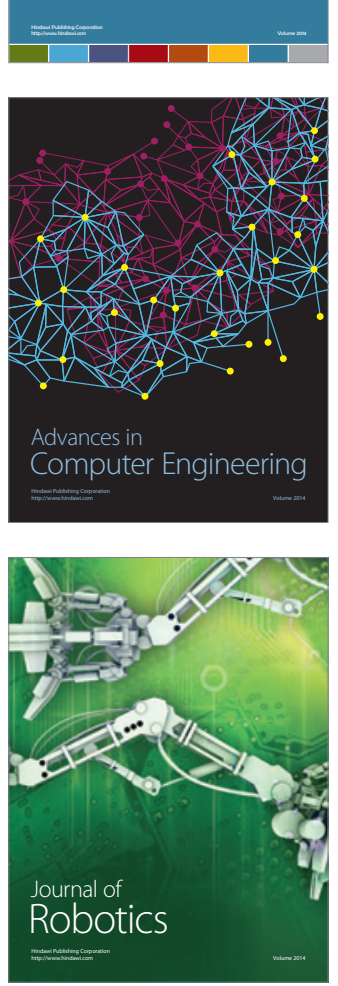
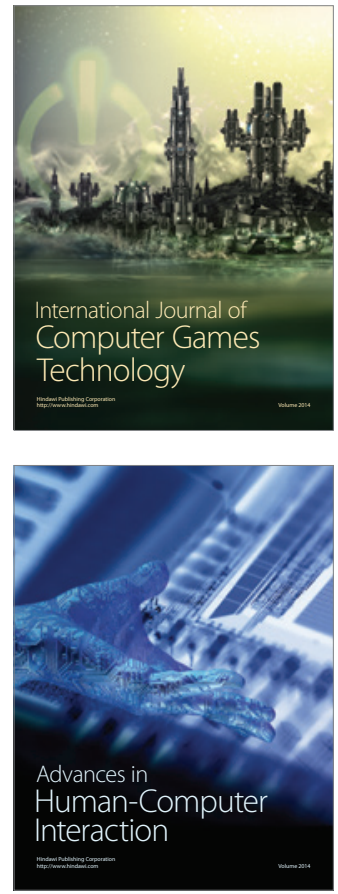
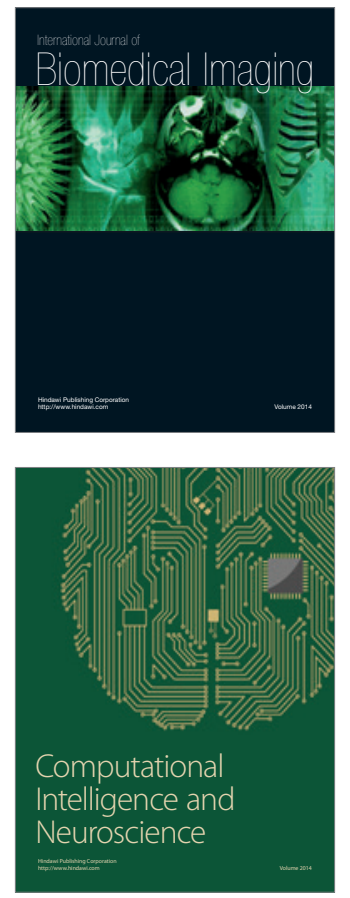
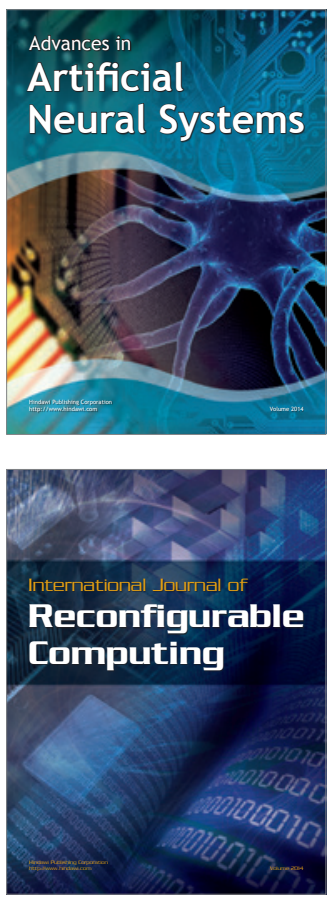
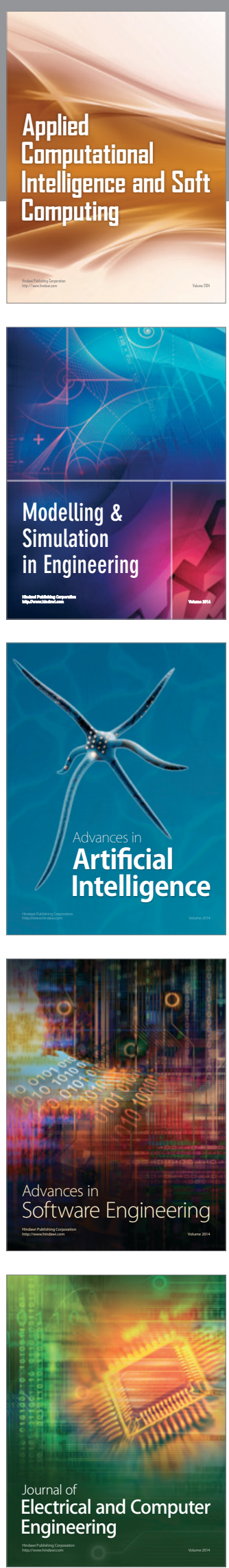